Received: 22 June 2018

Accepted: 9 July 2019

Published online: 23 July 2019

\section{A novel antibacterial resin-based root canal sealer modified by Dimethylaminododecyl Methacrylate}

Dan Liu ${ }^{1,2,4}$, Xian Peng ${ }^{1}$, Suping Wang ${ }^{1,2}$, Qi Han ${ }^{1,3}$, Bolei Li ${ }^{1,2}$, Xinxuan Zhou ${ }^{1}$, Biao Ren $\mathbb{1}^{1}$, Hockin H. K. Xu ${ }^{5}$, Michael D. Weir ${ }^{5}$, Mingyun $\mathrm{Li}^{1}$, Xuedong Zhou ${ }^{1,2}$ \& Lei Cheng ${ }^{1,2}$

Persistent apical periodontitis, mainly caused by microorganisms infections, represents a critical challenge for endodontists. Dimethylaminododecyl methacrylate (DMADDM) is a well-studied and potent antibacterial agent used in various studies described in the literature. The aim of this study is to develop a novel antibacterial root canal sealer by incorporating DMADDM into EndoREZ and investigate the properties of the resulting material. Different mass fractions $(0,1.25 \%, 2.5 \%$, and $5 \%)$ of DMADDM were incorporated into EndoREZ and the cytotoxicity, apical sealing ability and solubility of the resulting material were evaluated. Furthermore, a direct contact test, determination of colonyforming units, a crystal violet assay, scanning electronic microscopy and live/dead bacteria staining were performed to evaluate the antibacterial effect of the sealer to multispecies bacteria (Enterococcus faecalis, Streptococcus gordonii, Actinomyces naes(undii, and Lactobacillus acidophilus), in planktonic cells or biofilms. Fluorescence in situ hybridization and quantitative real-time polymerase chain reaction were carried out to assess the composition of the multispecies biofilms. No difference on the cytotoxicity, apical sealing ability and solubility between sealers containing DMADDM $(1.25 \%, 2.5 \%)$ and EndoREZ (0\%) could be determined. However, when the mass fraction of DMADDM increased to $5 \%$, significantly different properties were found compared to the $0 \%(p<0.05)$ group. Moreover, incorporating DMADDM into the sealer could greatly improve the antibacterial properties of EndoREZ. In addition, the composition ratio of $E$. faecalis could be decreased in multispecies microecology in sealers containing DMADDM. Therefore, a EndoREZ sealer material containing DMADDM could be considered useful in clinical applications for preventing and treating persistent apical periodontitis.

Persistent infections with microorganisms in the root canal represent the chief culprit to persistent apical periodontitis and endodontic failure ${ }^{1,2}$. However, the effective prevention and treatment for persistent apical periodontitis can not only rely on the development of new devices and technologies like microscopic root canal therapy, apical microsurgery ${ }^{3}$ or intentional replantation ${ }^{4}$, but further requires improving root filling materials, including root canal sealers and gutta percha. Root canal sealers play key roles in filling the space between core materials, or between core materials and root canal walls, or the space which core materials cannot reach, including lateral canals, apical ramification, furcation, etc ${ }^{5}$. Root canal sealers, with constant and effective antibacterial ability, have been shown to improve the success rate of endodontic treatments. Sealers exhibiting long-acting and potent antibacterial abilities can inhibit the growth of microorganisms, as well as reduce the occurrence of persisting bacterial after root canal filling. However, previous studies indicated that most of root canal sealers featured at

${ }^{1}$ State Key Laboratory of Oral Diseases \& National Clinical Research Center for Oral Diseases, West China Hospital of Stomatology Sichuan University, Chengdu, 610041, China. ${ }^{2}$ Department of Cariology and Endodontics, West China Hospital of Stomatology Sichuan University, Chengdu, 610041, China. ${ }^{3}$ Department of Oral Pathology, West China Hospital of Stomatology Sichuan University, Chengdu, 610041, China. ${ }^{4}$ Stomatological Hospital Affiliate to Zunyi Medical University, Zunyi Medical University, Zunyi, 563000, China. ${ }^{5}$ Department of Endodontics, Periodontics and Prosthodontics, University of Maryland Dental School, Baltimore, MD21201, USA. Correspondence and requests for materials should be addressed to M.L. (email: limingyun@scu.edu.cn) or Xuedong Zhou. (email: zhouxd@scu.edu. cn) or L.C. (email: chenglei@scu.edu.cn) 


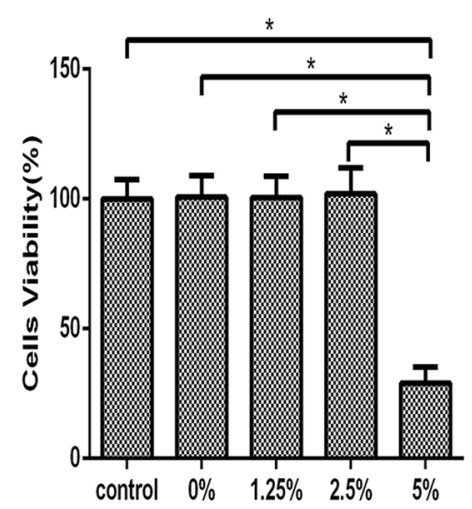

a

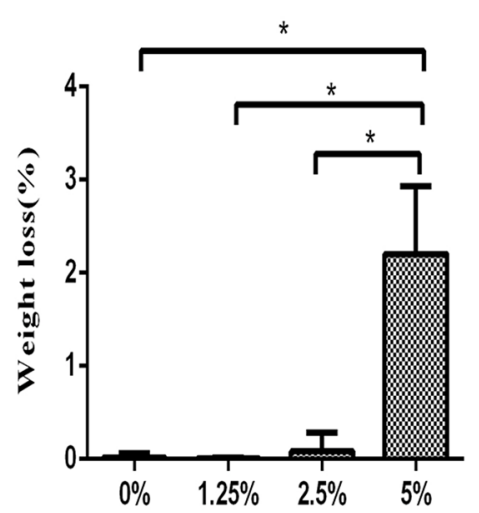

b

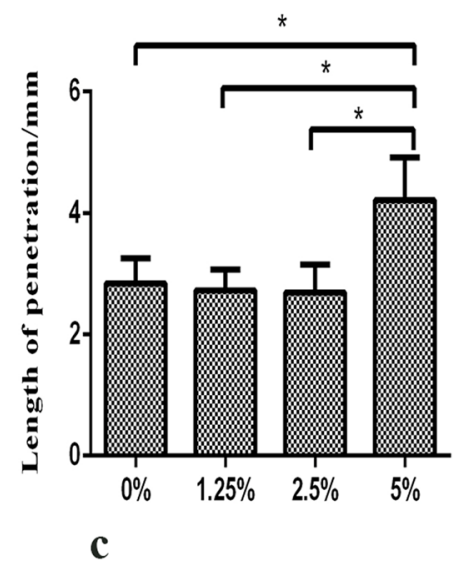

c
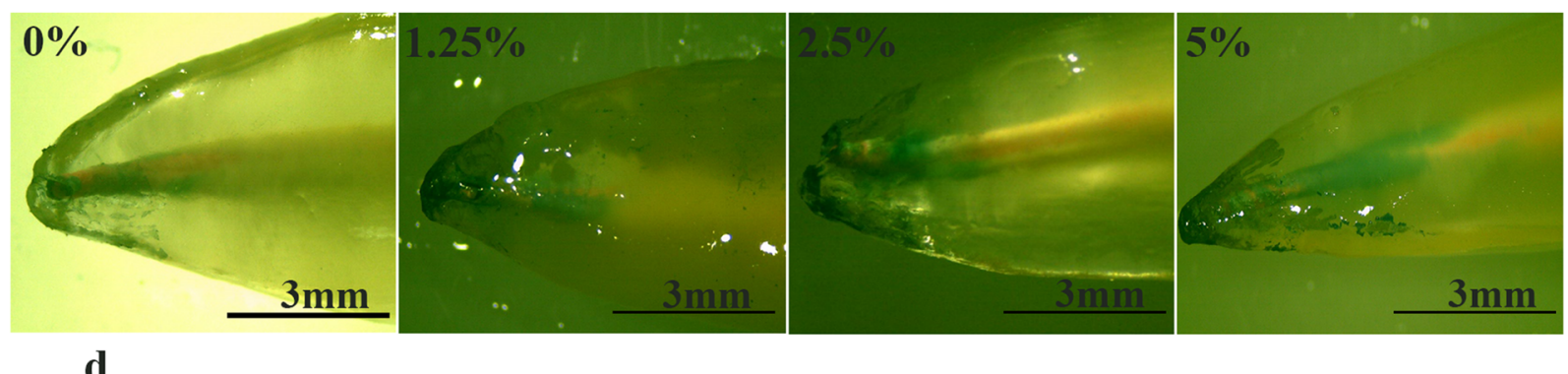

d

Figure 1. Correlation property tests of the EndoREZ containing different mass fractions of DMADDM ( $0 \%$, $1.25 \%, 2.5 \%, 5 \%)$. (a) Cytotoxicity assay of sealer eluents with mouse fibroblast. The control group contained pure culture medium. Each values is shown as mean $\pm \mathrm{SD}(n=6)\left({ }^{*} p<0.05\right)$; (b) Solubility test of sealers in different groups. Each values is shown as mean $\pm \mathrm{SD}(n=6)(* p<0.05)$; (c) Apical sealing ability test of the sealers. Each values is shown as mean $\pm \mathrm{SD}(n=6)(* p<0.05)$; (d) Images of apical sealing ability recorded using a stereomicroscope.

least some antibacterial activity when freshly prepared, and the antibacterial ability was lost as the materials set ${ }^{6}$. Various research groups have tried to add some antibacterial agents such as antibiotics ${ }^{7,8}$, nanomaterials ${ }^{7,9,10}$, quaternary ammonium salts ${ }^{10,11}$ into root canal sealer. But most of these studies only focused on one bacteria species, namely Enterococcus faecalis. Generally appreciated is the notion that chronic periapical disease or persistent periapical disease is caused by multispecies bacterial infections ${ }^{12}$. In addition, adding traditional antibiotics such as amoxicillin to sealers trigger general concerns of drug resistance. Meanwhile, it is often questionable whether the antibacterial agents added can react with the sealer and exhibit long-time potent antibacterial abilities. Last but not least, biocompatibility of the antibacterial-modified sealer is not mentioned in most of these studies found in the literature.

EndoREZ, the second generation of a methacrylate resin-based sealer, is a hydrophilic dual-cure urethane dimethacrylate (UDMA)-based material. It can flow into accessory canals and dentinal tubules to facilitate resin tag formation for retention and sealing after smear layer removal ${ }^{13}$. Nevertheless, the antibacterial ability of EndoREZ is quite weak ${ }^{14}$. Dimethylaminododecyl methacrylate (DMADDM) represents a tape of quaternary ammonium salts (QAS) and has been studied as an antibacterial agent ${ }^{15,16}$. In previous studies, it was shown that antibacterial QAS monomers can copolymerize with other monomers to form polymer matrices that can reduce bacterial growth ${ }^{17-19}$. Both chemical structures of the main components in EndoREZ and DMADDM feature double bonds that theoretically may form cross-link structures under certain conditions. Therefore, in this study, we intended to modify EndoREZ with DMADDM and investigate the sealer biocompatibility, relevant physicochemical properties, antibacterial efficiency and influence on the composition of multispecies- Enterococcus faecalis, Streptococcus gordonii, Actinomyces naeslundii, and Lactobacillus acidophilus.

\section{Results}

Cytotoxicity test and physical sealer properties. The results of the cytotoxicity test are shown in Fig. 1a. It was found that the EndoREZ was non-toxic to Mouse fibroblasts (L929). Moreover, adding DMADDM at a mass fraction of $1.25 \%$ or $2.5 \%$ did not increase cytotoxicity of the sealer. However, when the mass fraction of DMADDM increased to 5\%, the sealer cytotoxicity to L929 significantly increased. The solubility of the sealers in different group is shown in Fig. 1b. Though the samples were dried at $37^{\circ} \mathrm{C}$ for 14 days or 30 days, the weight of the samples in the groups containing $0 \%, 1.25 \%$, or $2.5 \%$ of DMADDM did not decrease even after immersion for 14 days. However, the solubility of the sealer in the group containing 5\% of DMADDM was significantly increased compared to the other groups $(p<0.05)$. There was no significant difference for the depth of dye penetration 


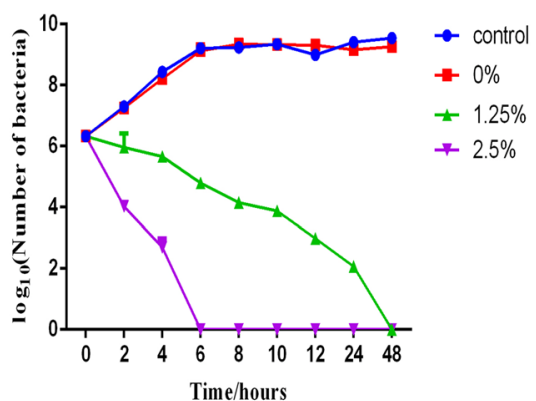

a
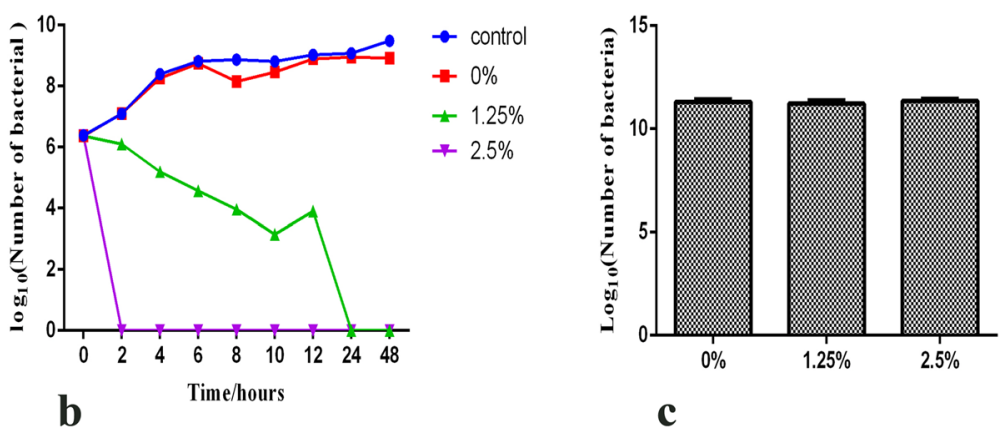

Figure 2. Antibacterial ability assays of the sealers containing different mass fractions of DMADDM ( $0 \%$, $1.25 \%, 2.5 \%$ ) after addition to multispecies planktonic bacteria. (a) Antibacterial ability of freshly prepared sealers. The control group consisted of primary bacteria suspension without any sealers. Each values is shown as mean $\pm \mathrm{SD}(n=4)$; (b) Antibacterial activity of the sealers set for 10 days and the control group consisting of pure bacteria suspension. Each values is shown as mean $\pm \mathrm{SD}(n=4)$; (c) Antibacterial efficiency of the sealer eluents, set for 10 days, added to multispecies after contacting for $24 \mathrm{~h}$ and $48 \mathrm{~h}$. Each values is shown as mean \pm $\mathrm{SD}(n=6)(* p<0.05)$.

(Fig. 1c,d) between EndoREZ and the sealers containing DMADDM at a mass fraction of $1.25 \%$ or $2.5 \%(p>0.1)$. However, the apical sealing ability of the group containing $5 \%$ of DMADDM was significantly weaker than that of other groups $(p<0.05)$. Therefore, according to the sealer biocompatibility results, including solubility and apical sealing ability, we included in our following experiments: 0\% DMADDM group, 1.25\% DMADDM group and $2.5 \%$ DMADDM group.

Antibacterial efficiency to planktonic bacteria. The results of the direct contact test are presented in Fig. 2a,b. Inspection of Fig. 2a shows that there was no antibacterial effect of the EndoREZ (0\% DMADDM group). Conversely, the sealers containing $1.25 \%$ and $2.5 \%$ of DMADDM presented a constant and effective antibacterial ability. Even after the sealers set for 10 days (Fig. 2b), no reduction of the antibacterial efficiency of the sealers containing DMADDM was found, demonstrating that addition of DMADDM to EndoREZ could add a long-time antibacterial ability to the sealer material. No antibacterial ability of the sealers eluent was seen $(p>0.1)$ (Fig. 2c), indirectly indicating that the monomer of DMADDM did not dissolve after mixing with EndoREZ.

Antibacterial effect on multispecies biofilms. Colony-forming units (CFU) (Fig. 3a) counting in the $1.25 \%, 2.5 \%$ DMADDM groups were an order of magnitude less than that in the $0 \%$ DMADDM group $(p<0.05)$. The biomass of the biofilms (Fig. $3 \mathrm{~b}$ ) in the $1.25 \%$ and $2.5 \%$ groups were found to be reduced compared to that of the control group ( $0 \%$ DMADDM) $(p<0.05)$, demonstrating that the addition of DMADDM to EndoREZ did inhibit the biofilm formation on the surface of the sealer. The scanning electronic microscope (SEM) images in different groups are shown in Fig. 3c. Meanwhile, the biofilm of the 0\% DMADDM group was much denser than the biofilm of the other two groups (1.25\% and 2.5\% of DMADDM). Figure $3 \mathrm{~d}$ shows live/dead staining confocal images of biofilms on sealers after $48 \mathrm{~h}$. Virtually full coverage of live bacteria could be determined in the EndoREZ group. However, the biofilms were found to be reduced in the $1.25 \%$ and $2.5 \%$ DMADDM groups compared to the EndoREZ group.

Composition ratio of bacteria in multispecies biofilm. The ratio variation of the bacteria in multispecies biofilm affected by EndoREZ with different concentrations of DMADDM is shown in Fig. $4 \mathrm{a}$. In the $0 \%$ DMADDM group, the proportion of $E$. faecalis reached a value of $31.42 \%$ and decreased continuously in all DMADDM groups, i.e. $24.85 \%$ and $23.26 \%$ respectively. The proportions of the other three bacteria species demonstrated slight increases. The result of fluorescence in situ hybridization is shown in Fig. 4b. S. gordonii was stained green, A. naeslundii and E. faecalis were stained blue, L. acidophilus was stained red, the probe of $L$. acidophilus (red) also showed a positive signal for $E$. faecalis. Because of this single mismatch within the binding site of this probe in E. faecalis, the simultaneous application of a second probe allowed for the identification of $E$. faecalis via a violet color. This combination of the probes was used to distinguish L.acidophilus and E. faecalis in the mixed community. The ratio of E. faecalis in the DMADDM groups was obviously lower than the ratio in the EndoREZ group.

\section{Discussion}

Microbes are considered to play the main etiological role of endodontic disease formation. The antibacterial activity of root canal sealers could help eliminate residual microorganisms. These novel antibacterial materials may be useful complementary to chemo-mechanical preparations and intracanal medication during root canal therapy. DMADDM, a quaternary ammonium monomer, was synthesized and demonstrated to be a strong antibacterial agent in various studies reported in the literature ${ }^{20-23}$. In the present study, we chose DMADDM as an antibacterial agent to modify the EndoREZ sealer and investigated the resulting antibacterial effect. The result of the direct contact test indicated that EndoREZ sealer alone exhibited no antibacterial effect on the multispecies biofilms consisting of E. faecalis, S. gordonii, A. naeslundii, and L. acidophilus. Our results were consistent with 

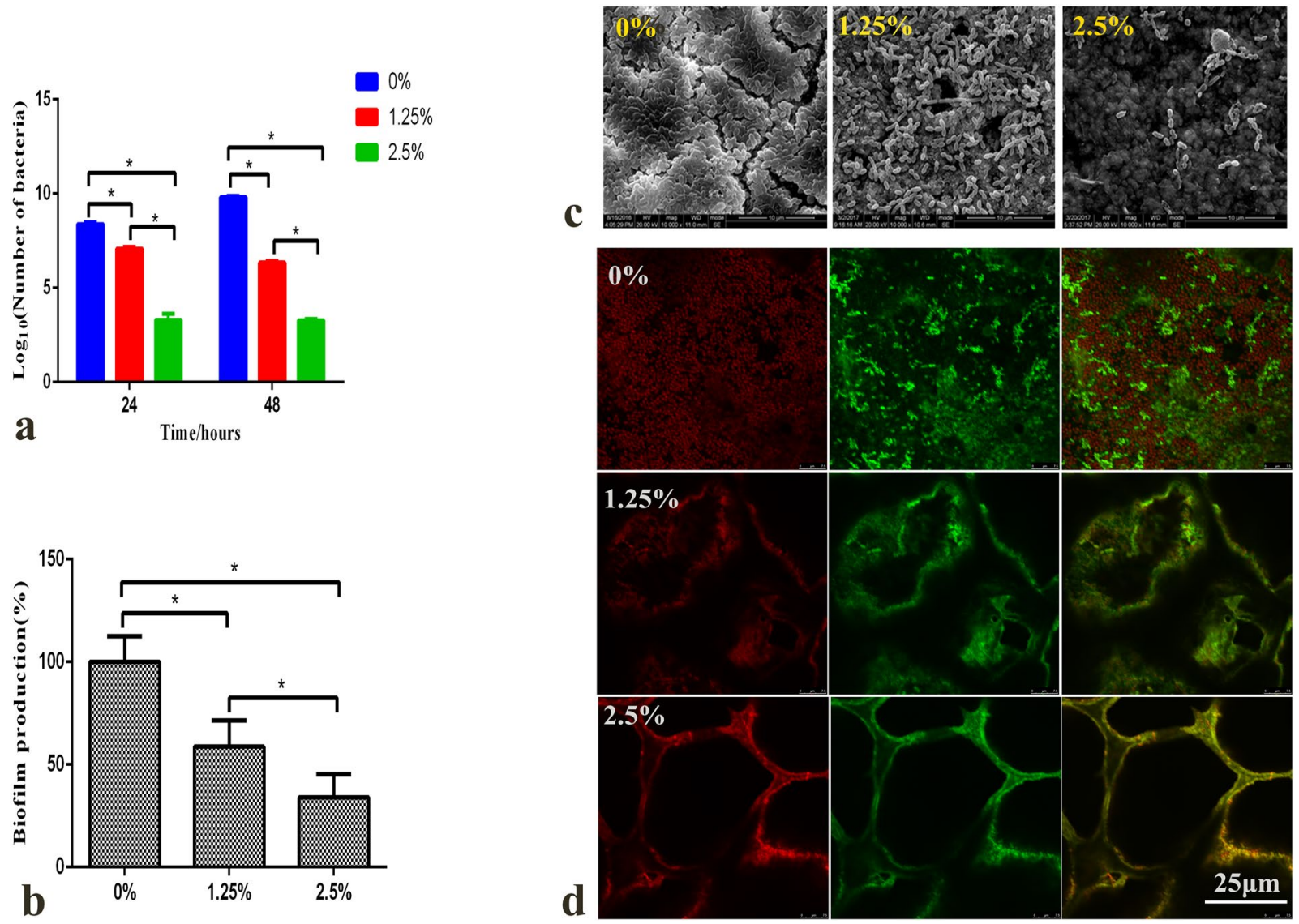

d

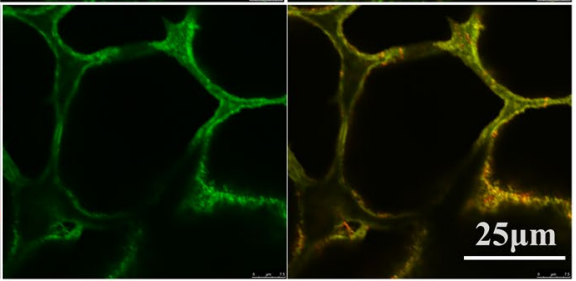

Figure 3. Antibacterial effect of the sealers containing different mass fractions of DMADDM (0\%, $1.25 \%$, $2.5 \%$ ) after addition to multispecies biofilms. (a) Colony-forming unit counts of biofilms formed on each disk after $24 \mathrm{~h}$ and $48 \mathrm{~h}$ from 3 groups containing $0 \%, 1.25 \%$, and 2.5\% DMADDM. Each values is shown as mean $\pm \mathrm{SD}(n=6)(* p<0.05)$; (b) Biofilms formation on different groups after $48 \mathrm{~h}$, tested via crystal violet assay. Each values is shown as mean $\pm \mathrm{SD}(n=6)(* p<0.05)$; (c) Scanning electron microscopy (SEM) images of multispecies biofilms; (d) Images of multispecies biofilms (live bacteria - stained green; dead cells - stained red) in different groups.

previously reported studies ${ }^{24,25}$, which showed that this methacrylate resin-based sealer had no antibacterial activity against $E$. faecalis. Conversely, the sealers containing both $1.5 \%$ and $2.5 \%$ mass fractions of DMADDM could significantly decrease the growth of the listed multispecies, no matter of the condition of the planktonic bacteria or biofilms. In addition, the antibacterial ability of the sealers containing DMADDM was not compromised, even after setting. Materials that feature some antimicrobial properties might gradually lose their volume, thus impairing the overall quality of the seal. However, the results of the solubility tests and antibacterial ability of the sealer eluent showed that the DMADDM antibacterial-modified sealers did not lose their volume and further exhibited an effective antibacterial behavior.

In root canal infections, bacteriological studies using various techniques have identified a selected number of bacteria that persist even after root canal treatment. These bacteria include mainly gram-positive facultative anaerobic bacteria, including enterococci, streptococci, lactobacilli, and actinomyces ${ }^{26}$. However, most previous studies about endodontic antibacterial efficiency of sealers mainly focused on one single species bacteria, namely E. faecalis ${ }^{27,28}$. In fact, persistent periapical diseases are often caused by multispecies bacteria. In a previous study, researchers have demonstrated that A. naeslundii, L. salivarius, S. gordonii, and E. faecalis can form stable and reproducible biofilm communities ${ }^{26}$. Besides, some studies indicated that E. faecalis was more resistant to starvation when found in coexistence with Candida albicans, S. gordonii, A. viscosus, or L. acidophilus ${ }^{12}$. Therefore, in our present study, we selected the species E. faecalis, S. gordonii, A. naeslundii, and L. acidophilus to form a multispecies and investigated the antibacterial efficiency of the sealer materials.

E. faecalis is the most frequently detected bacteria species in root canal-treated teeth. The species has been shown to be able to adapt to harsh environmental changes, such as an extreme alkaline $\mathrm{pH}$, salt concentrations, deprivation of nutrition, antimicrobial resistance, and grows in the root canal as a biofilm ${ }^{12,29}$. Although E. faecalis can be frequently found in root-filled teeth with apical periodontitis, in most cases, the strains were not isolated as a mono-infection. However, the ratio of E. faecalis in multispecies biofilms is considered an important index to evaluate the risk of apical periodontitis. Therefore, in the present study, a quantitative real-time polymerase chain reaction was performed to determine whether DMADDM could influence the bacterial ratio of biofilms. In doing so, we found that the sealers containing DMADDM could regulate the composition ratio of the multispecies 


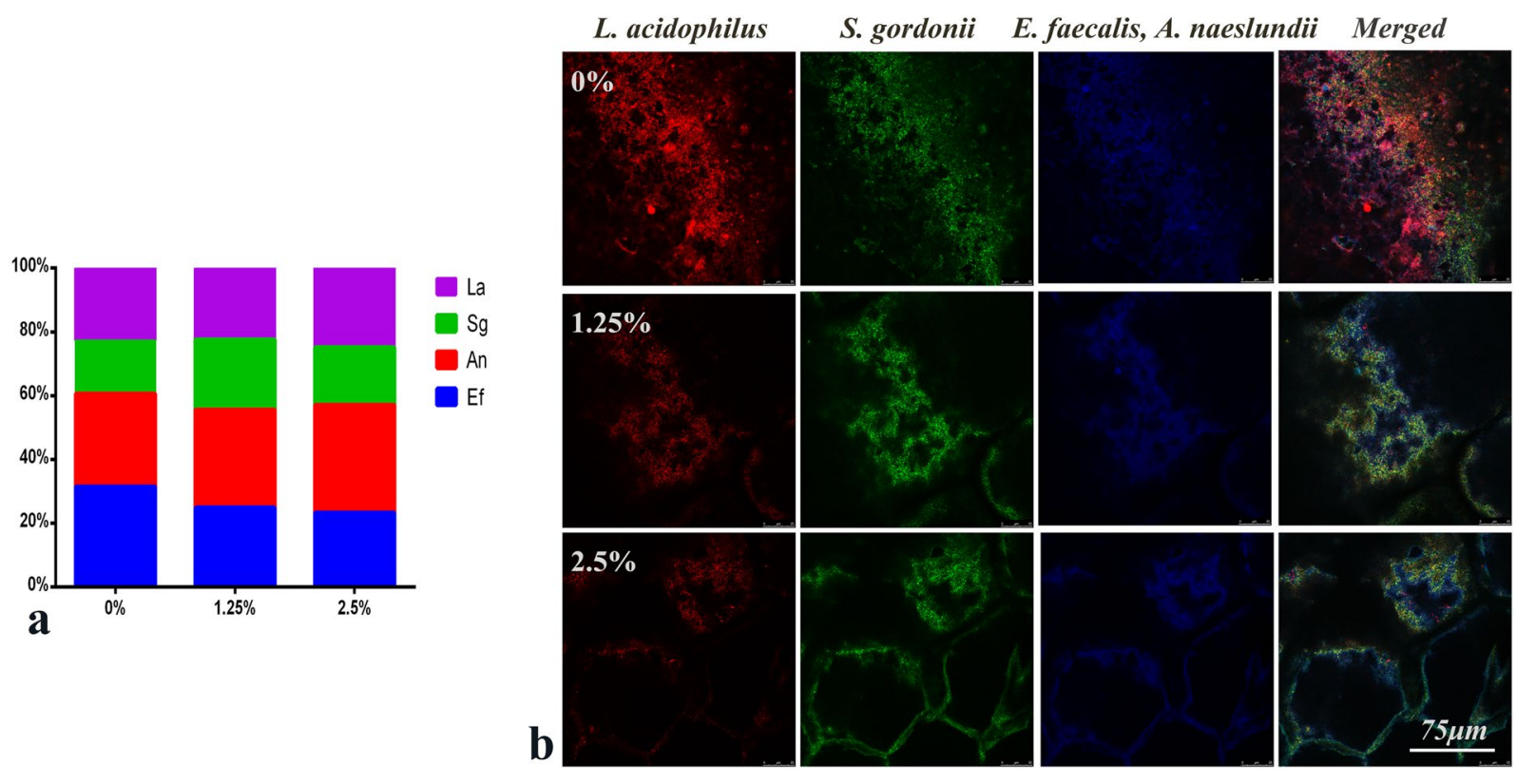

Figure 4. Ratios of four bacteria species in multispecies biofilms formed on the sealers containing different mass fractions of DMADDM $(0 \%, 1.25 \%, 2.5 \%)$. (a) Ratios of E. faecalis, S. gordonii, A. naeslundii, and $L$. acidophilus in multispecies biofilms, subjected to TaqMan real-time polymerase chain reaction; (b) Fluorescent in situ hybridization images of multispecies biofilms (S. gordonii - stained green; L. acidophilus - stained red; $E$. faecalis, A. naeslundii - stained blue). L. acidophilus (red) also gave a positive signal for E. faecalis, however, the latter could be more clearly identified by violet color staining.

biofilm and the presence of E. faecalis in the multispecies biofilms deceased upon increasing of the mass fractions of DMADDM in the sealer.

We have aslo tested the cytotoxicity of the sealer materials to ensure biocompatibility. Moreover, we have have investigated apical sealing ability to ensure that the addition of DMADDM to EndoREZ did not influence the sealers apical sealing ability. Finally, we have studied the solubility of the sealers to ensure that the quaternary ammonium monomer could not be released after mixing with EndoREZ. The results of the sealer eluent cytotoxicity, apical sealing ability and solubility were all similar. Adding DMADDM at a mass fraction of 5\% had a significant influence on the sealer cytotoxicity, apical sealing ability and solubility compared with the other groups containing less DMADDM. We hypothesized that when the mass fraction of DMADDM was increased to 5\%, a portion of DMADDM reacted with EndoREZ with the excess DMADDM portion still remaining anreacted. Hence, when the samples of group 5\% DADDM group were immersed in cell culture medium or distilled water, free DMADDM could leak out. This may be the reason for the corresponding cytotoxicity results of the sealer eluent, the apical sealing ability and solubility of the 5\% DMADDM group had a significant effect in contrast with other groups.

Using scanning electron microscopy (SEM) and LIVE/DEAD staining, we have observed the growth of biofilms on the surface of the sealers. In doing so, we have detected the distribution of the four bacteria species on the surface of different groups of sealers by fluorescence in situ hybridization (FISH). All images of the sealers containing DMADDM were different from that of the EndoREZ group, even in the group of sealers containing no bacteria (see Appendix). The amount of microorganisms on the surface of the experimental group was much less than that in the control group (0\% DMADDM). Furthermore, a structure-like grid on the surface of the sealers containing DMADDM could be seen, however, not in the group containing 0\% DMADDM. One explanation could be the formation of cross-links as a result of chemical reactions between DMADDM and EndoREZ. However, further studies are needed to confirm this hypothesis.

In conclusion, upon incorporating DMADDM (1.25\% or 2.5\%) to EndoREZ, a greatly improved long-term antibacterial ability of EndoREZ without compromising the biocompatibility, apical sealing ability and solubility of the sealers could be observed. Potentially, the incorporation of DMADDM could be an alternative to antibacterial sealers, ultimately improving periapical healing in endodontic treatments.

\section{Materials and Methods}

Ethical considerations. The study was conducted in accordance with the Declaration of Helsinki, the policy of Sichuan University and West China School of Stomatology. The protocol was approved by the Ethical Committee of West China School of Stomatology, Sichuan University (Chengdu, China) (Project identification code: WCHSIRB-D-2017-063, approval date: 02/03/2017). Written informed consent was obtained from each participant upon acquiring samples.

Bacteria species. Enterococcus faecalis ATCC29212, Streptococcus gordonii ATCC35105, Actinomyces naeslundii ATCC12104, and Lactobacillus acidophilus ATCC4356 were provided by the State Key Laboratory of 
Oral Diseases (Sichuan University, Chengdu, China). The bacteria were cultured in brain-heart infusion broth (BHI; Difco, Sparks, MD, USA), anaerobically $\left(90 \% \mathrm{~N}_{2}, 5 \% \mathrm{CO}_{2}, 5 \% \mathrm{H}_{2}\right)$ at $37^{\circ} \mathrm{C}$.

Synthesis of DMADDM and specimen preparation. DMADDM was synthesized and verified according to a method described previously ${ }^{30}$. DMADDM was mixed with EndoREZ (Ultradent, south Jordan, UT, USA), at a DMADDM (EndoREZ+DMADDM) mass fraction of $0 \%, 1.25 \%, 2.5 \%$ and $5 \%$, respectively.

Cytotoxicity of sealer eluent to mouse fibroblasts (L929). The biocompatibility of the sealer was determined through testing the cytotoxicity of the sealers eluent to mouse fibroblasts. Sealers containing different mass fractions of DMADDM $(0 \%, 1.25 \%, 2.5 \%, 5 \%)$ were injected to nonreactive plastic rings ( $5 \mathrm{~mm}$ in diameter and $2.5 \mathrm{~mm}$ in height), with a glass slide at the bottom using a $2-\mathrm{mL}$ syringe. The samples were completely set for 10 days at $100 \%$ humidity and at $37^{\circ} \mathrm{C}$. Then, the samples were sterilized in an ethylene oxide sterilizer (Anprolene AN 74i, Andersen, Haw River, NC, Germany) and immersed in $10 \mathrm{~mL}$ Dulbecco's Modified Eagle Medium (DMEM), 2\% fetal bovine serum, $100 \mathrm{IU} / \mathrm{mL}$ penicillin, and $100 \mathrm{IU} / \mathrm{mL}$ streptomycin ${ }^{31}$. The samples were then agitated for $24 \mathrm{~h}$ at $37^{\circ} \mathrm{C}$ to obtain the sealer eluents.

The cell line of mouse fibroblasts (L929) was cultured with $5 \% \mathrm{CO}_{2}$ at $37^{\circ} \mathrm{C}$. The cell Counting Kit-8 (DOjinDO, Shanghai, China) was used to assess the cytotoxicity of the eluent. 4000 cells/well were placed in 96-well plates (Costar, Corning Inc., Corning, NY, USA) and incubated for $24 \mathrm{~h}$ at $37^{\circ} \mathrm{C}$ in $5 \% \mathrm{CO}_{2}$ atmosphere. Then, the culture medium was discarded and replaced with $100 \mu \mathrm{L}$ of the sealers eluent. The cells were then cultured for another $24 \mathrm{~h}$. After removal of the culture medium, $100 \mu \mathrm{L}$ of fresh culture medium and $10 \mu \mathrm{L}$ of etrazolium-8-[2-(2-methoxy-4-nitrophenyl)-3-(4-nitrophenyl)-5-(2,4-disulfophenyl)-2H-tetrazolium] monosodium salt (CCK-8 solution) were added to each well. After incubation for another hour, the solution absorbance was measured at $450 \mathrm{~nm}$ using a Thermo Scientific Multiskan GO reader (Thermo Fisher Scientific Inc., Waltham, MA, USA) reader. The medium without eluent culturing L929 was used as control and each group contained 6 samples.

Apical sealing ability. Combined were the method of dye leakage with a tooth-clearing technique to evaluate the sealing property of the sealer ${ }^{32,33}$. Twenty four single rooted human anterior teeth with mature apex were selected from patients suffering from severe periodontitis or undergoing orthodontic treatment and the informed consent of the donors was obtained using a protocol approved by the West China Hospital of Stomatology, in Sichuan University. Routine access openings were prepared and then the working length was visually determined by subtracting $1 \mathrm{~mm}$ from the length of a size $10 \mathrm{~K}$-file at the apical foramen. All of the canals were instrumented to a working length with a size $40 \mathrm{~K}$-file using a step-back technique. The middle and coronal thirds were prepared using size 1-3 Gates-Glidden burs with a low-speed handpiece. The root canals were irrigated with $1 \mathrm{~mL}$ of $5.25 \% \mathrm{NaOCl}$ while instrumenting the canals. The patency of the apical foramen was confirmed with a size 10-15 K-files. Following root canal preparation, the canals were irrigated with $10 \mathrm{~mL}$ of $17 \%$ ethylenediaminetetraacetic acid (EDTA) for $1 \mathrm{~min}$ and $10 \mathrm{~mL}$ of $5.25 \% \mathrm{NaOCl}$. A syringe with a 23-gauge needle was used for irrigation. Finally, the root canals were flushed with $3 \mathrm{~mL}$ of distilled water and then dried with sterile paper points. The prepared teeth were randomly divided into 4 groups of 6 teeth each. The technique of lateral condensed gutta-percha using with sealers of different DMADDM groups was used to obturate the prepared root canals. Briefly, the canal walls were coated slightly with sealer using a size $35 \mathrm{~K}$-file and counter-clockwise motion. The apical part of the master gutta-percha cone was coated with the sealer from different DMADDM groups and placed into the canal. The root canals were filled with accessory cones by lateral condensation technique. The access openings were closed with light-cure flowable resin ( $3 \mathrm{M}$, Germany) and all specimens were kept in $100 \%$ humidity at $37^{\circ} \mathrm{C}$ for 7 days to allow for the sealer to set. The root surfaces of all specimens were prepared as reported previously ${ }^{34}$. Briefly, the root surfaces of all specimens were coated with two layers of nail varnish and sticky wax with the exception of the apical $1 \mathrm{~mm}$ specimen. The specimens were then immersed in $2 \%$ methylene blue solution for another 7 days at $37^{\circ} \mathrm{C}$. After seven days, the specimens were washed with tap water and dried, and the nail varnish and sticky wax were removed with a scalpel. The samples were then demineralized in $5 \%$ nitric acid for $72 \mathrm{~h}$ and the solution was changed every $24 \mathrm{~h}$. The specimens were rinsed with running tap water for $2 \mathrm{~h}$, dehydrated in ascending concentrations of $80 \%$ ethanol for $12 \mathrm{~h}, 90 \%$ and $100 \%$ ethanol for $24 \mathrm{~h}$ and finally cleared and stored in methyl salicylate. Linear dye penetration was determined using a stereomicroscope (Leica, Wetzlar, Germany) with electronic Vernier caliper. All procedures were performed by the same researcher.

Evaluation of solubility. Weight loss of the specimens was recorded as the sealer solubility ${ }^{35,36}$. The samples were prepared using the same method as in the cytotoxicity experiment described above. Each 10-day set sample was weighed three times in each group $(n=6)$ and recorded as W1. The samples were immersed in tubes containing $10 \mathrm{~mL}$ of distilled water for 14 days and then incubated for another 14 days at $37^{\circ} \mathrm{C}$ to dry the samples. Each sample was weighed 3 times and the weight was recorded as W2. The solubility (S) was calculated using the following formula: $\mathrm{S}=(\mathrm{W} 1-\mathrm{W} 2) / \mathrm{W} 1 \times 100 \%$.

Direct contact test. The antibacterial efficiency of the sealer was evaluated by means of a direct contact test described previously in the literature ${ }^{37} .20 \mathrm{mg}$ of the sealers were placed into 48 -well plates (Costar, Corning Inc., Corning, NY, USA) using a 2 -mL syringe. The fresh samples and 10-day set samples were prepared and sterilized using an ethylene oxide sterilizer. Then, $1 \mathrm{~mL}$ mixed inoculum containing E. faecalis $\left(1 \times 10^{6}\right.$ colony-forming units [CFUs] $/ \mathrm{mL})$, S. gordonii $\left(1 \times 10^{6} \mathrm{CFUs} / \mathrm{mL}\right)$, A. naeslundii $\left(1 \times 10^{6} \mathrm{CFUs} / \mathrm{mL}\right)$, and L. acidophilus $\left(1 \times 10^{6}\right.$ CFUs $/ \mathrm{mL}$ ), was added to each well. The well without any sealer served as negative control. The plates were incubated at $37^{\circ} \mathrm{C}$ anaerobically $\left(90 \% \mathrm{~N}_{2}, 5 \% \mathrm{CO}_{2}, 5 \% \mathrm{H}_{2}\right)$. After cultured duration for $0 \mathrm{~h}, 2 \mathrm{~h}, 4 \mathrm{~h}, 6 \mathrm{~h}, 8 \mathrm{~h}$, $10 \mathrm{~h}, 12 \mathrm{~h}, 24 \mathrm{~h}$ and $48 \mathrm{~h}, \mathrm{BHI}$ agar plates were used to assess the microorganism viability after serial dilution in phosphate-buffered saline (PBS). 
Antibacterial efficiency of the sealers eluent. In order to determine the antibacterial efficiency of the sealers eluent, $20 \mathrm{mg}$ of the sealers were placed on 48 -well plates using a 2 -mL syringe. The samples were then set for 10 days at $100 \%$ humidity and sterilized using an ethylene oxide sterilizer. Then, $1 \mathrm{~mL}$ of brain heart infusion (BHI) broth was added to each well and the 48 -well plates were agitated for $24 \mathrm{~h}$ at $37^{\circ} \mathrm{C}$ to obtain the sealers eluent. Subsequently, $500 \mu \mathrm{L}$ of the sealers eluent was transferred to a new 48 -well plate and each well contained $500 \mu \mathrm{L}$ of mixed bacterial inoculum, containing a defined microbial population consisting of E. faecalis $\left(1 \times 10^{6}\right.$ CFUs/mL), S. gordonii $\left(1 \times 10^{6} \mathrm{CFUs} / \mathrm{mL}\right)$, A. naeslundii $\left(1 \times 10^{6} \mathrm{CFUs} / \mathrm{mL}\right)$, and L. acidophilus $\left(1 \times 10^{6} \mathrm{CFUs} /\right.$ $\mathrm{mL})$. Next, the 48 -well plates were incubated in an anaerobic chamber for $48 \mathrm{~h}$. BHI agar plates were used to assess the microorganism viability after serial dilution in PBS.

Fabrication of biofilm specimens. The specimens for the biofilm experiments were prepared following a study described previously in the literature ${ }^{38}$. Briefly, composite disks were fabricated using the cover of a sterile 48-well plate as a mold. $20 \mathrm{mg}$ of the sealers containing DMADDM or the control sealer were applied on the surface of each composite disk and flatted using a spatula. The specimens were then placed into a 24 -well plate and incubated at $37^{\circ} \mathrm{C}$ with $100 \%$ humidity for 10 days. Next, the samples were sterilized in an ethylene oxide sterilizer. For multispecies biofilm formation, bacterial suspensions were mixed to obtain an inoculum containing a defined microbial population consisting of E. faecalis $\left(1 \times 10^{6} \mathrm{CFUs} / \mathrm{mL}\right), S$. gordonii $\left(1 \times 10^{6} \mathrm{CFUs} / \mathrm{mL}\right), A$. naeslundii $\left(1 \times 10^{6} \mathrm{CFUs} / \mathrm{mL}\right)$, and L. acidophilus $\left(1 \times 10^{6} \mathrm{CFUs} / \mathrm{mL}\right)$ in $2 \mathrm{~mL}$ of BHI with $1 \%$ sucrose for $24 \mathrm{~h}$, and $48 \mathrm{~h}$ at $37^{\circ} \mathrm{C}$ anaerobically to form biofilms.

Colony-forming units (CFU). After cultured for $24 \mathrm{~h}$ and $48 \mathrm{~h}$, the biofilms were gently rinsed twice with phosphate buffered saline (PBS) to remove the planktonic bacteria. The biofilms in each group were then harvested by scraping and sonication in PBS buffer. After preparation of serial dilutions in PBS, the bacteria were incubated on the BHI agar plates to count microorganism colonies and assess the viability.

Crystal violet assay. The crystal violet assay used in this study was carried out in accordance with a study published previously ${ }^{39}$. Briefly, disks with multispecies biofilms after $48 \mathrm{~h}$ were washed twice with PBS and fixed for 15 minutes using $99 \%$ methanol. Then, the well contents were aspirated and the plates were allowed to dry. The biofilms were stained with $1 \mathrm{~mL} 0.1 \%$ crystal violet solution for $5 \mathrm{~min}$. Excess stain was gently rinsed off with tap water and the plates were dried thereafter. The obtained stain was resolubilized in $1 \mathrm{~mL}$ of $95 \%$ ethanol with shaking in an orbital shaker for $30 \mathrm{~min}$, and the ethanol was transferred to a new 96-well-plate. The solution absorbance was then measured using a Thermo Scientific Multiskan GO reader (Thermo Fisher Scientific Inc., Waltham, MA, USA) at $595 \mathrm{~nm}$.

Biofilm structure detected by scanning electronic microscopy. Specimens for scanning electronic microscopy were prepared as described above. The disks with $48 \mathrm{~h}$ biofilms were gently washed twice with $2 \mathrm{~mL}$ of PBS and fixed with $2 \mathrm{~mL}$ of $2.5 \%$ glutaraldehyde overnight. The specimens were rinsed with PBS and then subjected to graded-ethanol $(50 \%, 60 \%, 70 \%, 80 \%, 90 \%, 95 \%$, and $100 \%)$ dehydrations, with 15 minutes in different concentration of ethanol. The specimens were then sputter-coated with gold and examined by scanning electronic microscopy (SEM, Quanta 200, FEI, Hillsboro, OR, USA) ${ }^{40}$. Each group contained 6 samples and each biofilm was scanned in at least five randomly selected positions.

Live/dead bacteria staining. The biofilms after $48 \mathrm{~h}$ were washed twice with PBS and stained using the BacLight Live/Dead bacterial viability kit (Molecular Probes, Eugene, OR, USA) following the instructions provided by manufacturer ${ }^{41,42}$. Live bacteria were stained with Syto 9 and dead bacteria were stained with propidium iodide. Biofilm images were detected using a Leica DMIRE2 confocal laser scanning microscope (CLSM) (Leica, Wetzlar, Germany) equipped with a $63 \times(1.4$ numerical aperture $)$ oil immersion objective lens. Each biofilm was scanned in at least five randomly selected positions.

Fluorescent in situ hybridization (FISH). For FISH analysis of biofilm cells, the species-specific probes and the fabrication of the specimens were carried out according to a method described previously ${ }^{26}$. Briefly, the biofilms were fixed in $4 \%$ paraformaldehyde. The fixed disks were then washed with phosphate-buffered saline and stored at $4{ }^{\circ} \mathrm{C}$. Next, the fixed biofilms were permeabilized using lysozyme $(30 \mathrm{mg} / \mathrm{mL})$ in $100 \mathrm{mM}$ of Tris- $\mathrm{HCl}(\mathrm{pH}=7.5)$ and $50 \mathrm{mM}$ EDTA for 20 minutes at $37^{\circ} \mathrm{C}$. The biofilms were then washed with ultrapure water and dehydrated with 50\%, $80 \%$ and $96 \%$ ethanol for 3 minutes, respectively. The dehydrated disks were then inoculated with $30 \mathrm{~mL}$ hybridization buffer containing $20 \%$ formamide and the oligonucleotide probes. Each of the probes was then diluted in the hybridization buffer to an approximate concentration of $20 \mathrm{ng} / \mathrm{mL}$. After 90 minutes of hybridization at $46^{\circ} \mathrm{C}$, the hybridization buffer was removed, and the chambers were washed with washing buffer $(20 \mathrm{mM}$ Tris- $\mathrm{HCl}$ buffer, $\mathrm{pH}=7.5$, containing $5 \mathrm{mM}$ EDTA, $0.02 \%$ sodium dodecyl sulfate, and $220 \mathrm{mM} \mathrm{NaCl}$ ) for 15 minutes at $48^{\circ} \mathrm{C}$. Then, the chambers were rinsed with ultrapure water and the biofilms were imaged using a confocal laser scanning microscope (Leica, Wetzlar, Germany). Each biofilm was scanned in at least five randomly selected positions.

DNA isolation and quantitative real-time polymerase chain reaction. The total DNA of biofilms incubated for $48 \mathrm{~h}$ was isolated and purified using a Yeast/Bact Kit B (QIAGEN Science, Maryland, USA) following the instructions provided by manufacturer. The purity and concentration of DNA were detected using a NanoDrop 2000 spectrophotometer (Thermo Scientific, Waltham, MA, USA). Quantitative real-time PCR was used to quantify the absolute number of E. faecalis, S. gordonii, A. naeslundii, and L. acidophilus, performed on a C1000 Touch ${ }^{\mathrm{TM}}$ Thermal Cycler instrument (Bio-Rad, Philadelphia, PA, USA) with the SYBR reagent (Takara, Dalian, China). The BLAST tool on NCBI (http://blast.ncbi.nlm.nih.gov/Blast.cgi) was used to compare the 


\begin{tabular}{|l|l|l|}
\hline \multirow{2}{*}{ Bacteria } & $\begin{array}{l}\text { Sequence } \\
\left(\mathbf{5}^{\prime}-\mathbf{>} \mathbf{3}^{\prime}\right)\end{array}$ & Template strand \\
\hline \multirow{2}{*}{ E. faecalis } & $\mathrm{F}$ & ATTGGAAAGAGGAGTGGCGG \\
\cline { 2 - 3 } & $\mathrm{R}$ & TGAGCCGTTACCTCACCAAC \\
\hline \multirow{2}{*}{ S. gordonii } & $\mathrm{F}$ & GAGTGCTAGGTGTTAGGCCC \\
\cline { 2 - 3 } & $\mathrm{R}$ & CCTGGTAAGGTTCTTCGCGT \\
\hline \multirow{2}{*}{ A. naeslundii } & $\mathrm{F}$ & CTCGACACCGTGAAGTTGGA \\
\cline { 2 - 3 } & $\mathrm{R}$ & CGACTTCGTCCCAATCACCA \\
\hline \multirow{2}{*}{ L. acidophilus } & $\mathrm{F}$ & TGGGGAACCTGCCCCATAG \\
\cline { 2 - 3 } & $\mathrm{R}$ & GGTAGGCCGTTACCCTACCA \\
\hline
\end{tabular}

Table 1. Specific Primers used for qPCR.

genomes and to design species-specific primer. The sequences of the primers for the four bacteria species can be found listed in Table 1.

Statistical analysis. All the experiments were repeated at least 3 times independently. Statistical analysis was performed with the SPSS software, version 16.0 (SPSS Inc., Chicago, IL, USA). One-way analysis of variance and Student-Newman-Keuls test were used for all pairwise comparison. Significant differences were considered when $p<0.05$.

\section{Data Availability}

The datasets generated and analyzed during the current study are available from the corresponding author on reasonable requests.

\section{References}

1. Arnold, M., Ricucci, D. \& Siqueira, J. F. Jr. Infection in a complex network of apical ramifications as the cause of persistent apical periodontitis: a case report. J Endod 39, 1179-1184, https://doi.org/10.1016/j.joen.2013.04.036 (2013).

2. Nair, P. N. On the causes of persistent apical periodontitis: a review. Int Endod J 39, 249-281, https://doi.org/10.1111/j.13652591.2006.01099.x (2006).

3. Signoretti, F. G. et al. Persistent extraradicular infection in root-filled asymptomatic human tooth: scanning electron microscopic analysis and microbial investigation after apical microsurgery. J Endod 37, 1696-1700, https://doi.org/10.1016/j.joen.2011.09.018 (2011).

4. Cotter, M. R. \& Panzarino, J. Intentional replantation: a case report. J Endod 32, 579-582, https://doi.org/10.1016/j.joen.2005.08.004 (2006).

5. Balguerie, E., van der Sluis, L., Vallaeys, K., Gurgel-Georgelin, M. \& Diemer, F. Sealer penetration and adaptation in the dentinal tubules: a scanning electron microscopic study. J Endod 37, 1576-1579, https://doi.org/10.1016/j.joen.2011.07.005 (2011).

6. AlShwaimi, E. et al. In Vitro Antimicrobial Effectiveness of Root Canal Sealers against Enterococcus faecalis: A Systematic Review. J Endod 42, 1588-1597, https://doi.org/10.1016/j.joen.2016.08.001 (2016).

7. Kangarlou, A., Neshandar, R., Matini, N. \& Dianat, O. Antibacterial efficacy of AH Plus and AH26 sealers mixed with amoxicillin, triple antibiotic paste and nanosilver. Journal of dental research, dental clinics, dental prospects 10, 220-225, https://doi.org/10.15171/ joddd.2016.035 (2016).

8. Collares, F. M. et al. Methacrylate-based root canal sealer containing chlorexidine and alpha-tricalcium phosphate. Journal of biomedical materials research. Part B, Applied biomaterials, https://doi.org/10.1002/jbm.b.33946 (2017).

9. Del Carpio-Perochena, A., Kishen, A., Shrestha, A. \& Bramante, C. M. Antibacterial Properties Associated with Chitosan Nanoparticle Treatment on Root Dentin and 2 Types of Endodontic Sealers. J Endod 41, 1353-1358, https://doi.org/10.1016/j. joen.2015.03.020 (2015).

10. Barros, J. et al. Antibacterial, physicochemical and mechanical properties of endodontic sealers containing quaternary ammonium polyethylenimine nanoparticles. Int Endod J 47, 725-734, https://doi.org/10.1111/iej.12207 (2014).

11. Kitagawa, R. et al. Development of an antibacterial root canal filling system containing MDPB. Journal of dental research 93 , 1277-1282, https://doi.org/10.1177/0022034514549808 (2014).

12. Gao, Y., Jiang, X., Lin, D., Chen, Y. \& Tong, Z. The Starvation Resistance and Biofilm Formation of Enterococcus faecalis in Coexistence with Candida albicans, Streptococcus gordonii, Actinomyces viscosus, or Lactobacillus acidophilus. J Endod 42, 1233-1238, https://doi.org/10.1016/j.joen.2016.05.002 (2016).

13. Kim, Y. K. et al. Critical review on methacrylate resin-based root canal sealers. J Endod 36, 383-399, https://doi.org/10.1016/j. joen.2009.10.023 (2010)

14. Eldeniz, A. U., Erdemir, A., Hadimli, H. H., Belli, S. \& Erganis, O. Assessment of antibacterial activity of EndoREZ. Oral surgery, oral medicine, oral pathology, oral radiology, and endodontics 102, 119-126, https://doi.org/10.1016/j.tripleo.2005.06.017 (2006).

15. Zhang, K. et al. Effect of Antimicrobial Denture Base Resin on Multi-Species Biofilm Formation. International journal of molecular sciences 17, https://doi.org/10.3390/ijms17071033 (2016).

16. Cheng, L. et al. Nanotechnology strategies for antibacterial and remineralizing composites and adhesives to tackle dental caries. Nanomedicine 10, 627-641, https://doi.org/10.2217/nnm.14.191 (2015).

17. Cheng, L. et al. Antibacterial nanocomposite with calcium phosphate and quaternary ammonium. Journal of dental research $\mathbf{9 1}$, 460-466, https://doi.org/10.1177/0022034512440579 (2012).

18. Wang, S. et al. Antibacterial effect of dental adhesive containing dimethylaminododecyl methacrylate on the development of Streptococcus mutans biofilm. International journal of molecular sciences 15, 12791-12806, https://doi.org/10.3390/ijms150712791 (2014).

19. Cheng, L. et al. Developing a New Generation of Antimicrobial and Bioactive Dental Resins. Journal of dental research 96, 855-863, https://doi.org/10.1177/0022034517709739 (2017).

20. Chen, H. et al. Heat-Polymerized Resin Containing Dimethylaminododecyl Methacrylate Inhibits Candida albicans Biofilm. Materials (Basel) 10, https://doi.org/10.3390/ma10040431 (2017).

21. Zhou, W. et al. Novel Cavity Disinfectants Containing Quaternary Ammonium Monomer Dimethylaminododecyl Methacrylate. Materials (Basel) 9, https://doi.org/10.3390/ma9080674 (2016). 
22. Ge, Y. et al. The Use of Quaternary Ammonium to Combat Dental Caries. Materials 8, 3532-3549, https://doi.org/10.3390/ ma8063532 (2015).

23. Zhang, K. et al. Effect of antibacterial dental adhesive on multispecies biofilms formation. Journal of dental research 94, 622-629, https://doi.org/10.1177/0022034515571416 (2015).

24. Baer, J. \& Maki, J. S. In Vitro Evaluation of the Antimicrobial Effect of Three Endodontic Sealers Mixed with Amoxicillin. Journal Of Endodontics 36, 1170-1173, https://doi.org/10.1016/j.joen.2010.03.033 (2010).

25. Slutzky-Goldberg, I. et al. Antibacterial properties of four endodontic sealers. Journal Of Endodontics 34, 735-738, https://doi. org/10.1016/j.joen.2008.03.012 (2008).

26. Chavez de Paz, L. E. Development of a multispecies biofilm community by four root canal bacteria. J Endod 38, 318-323, https://doi. org/10.1016/j.joen.2011.11.008 (2012).

27. Barros, J. et al. Antibiofilm effects of endodontic sealers containing quaternary ammonium polyethylenimine nanoparticles. J Endod 40, 1167-1171, https://doi.org/10.1016/j.joen.2013.12.021 (2014).

28. Zhang, H., Shen, Y., Ruse, N. D. \& Haapasalo, M. Antibacterial activity of endodontic sealers by modified direct contact test against Enterococcus faecalis. J Endod 35, 1051-1055, https://doi.org/10.1016/j.joen.2009.04.022 (2009).

29. Stuart, C. H., Schwartz, S. A., Beeson, T. J. \& Owatz, C. B. Enterococcus faecalis: Its Role in Root Canal Treatment Failure and Current Concepts in Retreatment. Journal Of Endodontics 32, 93-98, https://doi.org/10.1016/j.joen.2005.10.049 (2006)

30. Zhou, C. et al. Synthesis of new antibacterial quaternary ammonium monomer for incorporation into CaP nanocomposite. Dental materials: official publication of the Academy of Dental Materials 29, 859-870, https://doi.org/10.1016/j.dental.2013.05.005 (2013).

31. Tai, K. W., Huang, F. M. \& Chang, Y. C. Cytotoxic evaluation of root canal filling materials on primary human oral fibroblast cultures and a permanent hamster cell line. J Endod 27, 571-573, https://doi.org/10.1097/00004770-200109000-00004 (2001).

32. Venturi, M., Prati, C., Capelli, G., Falconi, M. \& Breschi, L. A preliminary analysis of the morphology of lateralcanals after root canal filling using a tooth-clearingtechnique. International Endodontic Journal 36, 54-63 (2003).

33. Zmener, O., Pameijer, C. H. \& Macri, E. Evaluation of the apical seal in root canals prepared with a new rotary system and obturated with a methacrylate based endodontic sealer: an in vitro study. J Endod 31, 392-395 (2005).

34. Sevimay, S. \& Kalayci, A. Evaluation of apical sealing ability and adaptation to dentine of two resin-based sealers. Journal of oral rehabilitation 32, 105-110, https://doi.org/10.1111/j.1365-2842.2004.01385.x (2005)

35. Schäfer, E. \& Zandbiglari, T. Solubility of root-canal sealers in water andartificial saliva. International Endodontic Journal 36, 660-669 (2003).

36. Lim, E. S. et al. Physical properties and biocompatibility of an injectable calcium-silicate-based root canal sealer: in vitro and in vivo study. BMC oral health 15, 129, https://doi.org/10.1186/s12903-015-0112-9 (2015).

37. Heyder, M. et al. Antibacterial effect of different root canal sealers on three bacterial species. Dental materials: official publication of the Academy of Dental Materials 29, 542-549, https://doi.org/10.1016/j.dental.2013.02.007 (2013).

38. Li, F. et al. Effects of a dental adhesive incorporating antibacterial monomer on the growth, adherence and membrane integrity of Streptococcus mutans. J Dent 37, 289-296, https://doi.org/10.1016/j.jdent.2008.12.004 (2009).

39. Mataraci, E. \& Dosler, S. In vitro activities of antibiotics and antimicrobial cationic peptides alone and in combination against methicillin-resistant Staphylococcus aureus biofilms. Antimicrobial agents and chemotherapy 56, 6366-6371, https://doi. org/10.1128/AAC.01180-12 (2012).

40. Han, Q. et al. Anti-Caries Effects of Dental Adhesives Containing Quaternary Ammonium Methacrylates with Different Chain Lengths. Materials (Basel) 10, https://doi.org/10.3390/ma10060643 (2017).

41. Wang, S. P. et al. Effect of anti-biofilm glass-ionomer cement on Streptococcus mutans biofilms. International journal of oral science 8, 76-83, https://doi.org/10.1038/ijos.2015.55 (2016).

42. Cheng, L. et al. One-year water-ageing of calcium phosphate composite containing nano-silver and quaternary ammonium to inhibit biofilms. International journal of oral science 8, 172-181, https://doi.org/10.1038/ijos.2016.13 (2016).

\section{Acknowledgements}

This study was supported by National Key Research and Development Program of China [no. 2017YFC0840100 and 2017YFC0840107 (LC)], the National Natural Science Foundation of China [no. 81372889 (LC), 81430011 (XZ)], International Science and Technology Program of Sichuan [no. 2017HH0008 (LC)], the Innovative Research Team Program of Sichuan Province (LC), University of Maryland School of Dentistry Bridging Fund and Seed Grant (HX), A research grant from the Department of Science and Technology, Sichuan Province, 2016JY0006(XZ).

\section{Author Contributions}

Dan Liu, Mingyun Li, Xuedong Zhou and Lei Cheng conceived and designed the experiments; Dan Liu, Xian Peng, Suping Wang, Qi Han, Bolei Li, Xinxuan Zhou, Biao Ren, Mingyun Li performed the experiments; Dan Liu, Xian Peng, Suping Wang analyzed the data; Dan Liu, Mingyun Li and Lei Cheng wrote the paper; Hockin H. K. $\mathrm{Xu}$, Michael D and Xuedong Zhou revised the paper. All authors reviewed the manuscript.

\section{Additional Information}

Supplementary information accompanies this paper at https://doi.org/10.1038/s41598-019-47032-8.

Competing Interests: The authors declare no competing interests.

Publisher's note: Springer Nature remains neutral with regard to jurisdictional claims in published maps and institutional affiliations.

(c) (i) Open Access This article is licensed under a Creative Commons Attribution 4.0 International

License, which permits use, sharing, adaptation, distribution and reproduction in any medium or format, as long as you give appropriate credit to the original author(s) and the source, provide a link to the Creative Commons license, and indicate if changes were made. The images or other third party material in this article are included in the article's Creative Commons license, unless indicated otherwise in a credit line to the material. If material is not included in the article's Creative Commons license and your intended use is not permitted by statutory regulation or exceeds the permitted use, you will need to obtain permission directly from the copyright holder. To view a copy of this license, visit http://creativecommons.org/licenses/by/4.0/.

(c) The Author(s) 2019 